\title{
Open Piezo Preservation Rhinoplasty: A Case Report of the New Rhinoplasty Approach
}

\author{
Abdulkadir Goksel, MD ${ }^{1}$ Yves Saban, MD² \\ ${ }^{1}$ Rinolstanbul Clinic, İstanbul, Turkey \\ 2 Private Practice, Nice, France \\ Facial Plast Surg 2019;35:113-118.
}

\begin{abstract}
Address for correspondence Abdulkadir Goksel, MD, Rinolstanbul Clinic, Bağdat Cad. Zümrüt, Apt. No: 378 Kat:1 Daire:5, Şaşkınbakkal, istanbul, Turkey 34728 (e-mail: akgoksel@gmail.com).
\end{abstract}

Only a decade ago, surgeons could not imagine that there would be an opportunity to use atraumatic devices and reshape the bone without breaking it, to keep the ligaments, soft tissue, or skin attachments intact. Now we can declare that the rhinoplasty revolution has begun, proving it by preservation rhinoplasty results with faster recovery and more natural looks. Preservation of nasal dorsal anatomy does not disrupt the natural anatomy, and one can avoid many smaller and larger complications.

In this case report, we have performed an open approach preservation rhinoplasty by using Piezoelectric device. We preserved both scroll and Pitanguy's ligaments and did not dissect the skin and soft tissue on the dorsum and preserved it at the same time.

\section{Choosing the Patient}

When deciding whether to preserve the nasal dorsum or not, it is essential to access the patient's dorsum and its deformity. Also, the severity and composition of the dorsal convexity must be evaluated.

There are strict indications for maximal structure preserving on the dorsum. Appropriate candidates for this are primary patients who have good dorsal aesthetics and only need reduction of the dorsal profile line. In this group, it is possible to apply preservation rhinoplasty via an open approach with no skin elevation on the dorsum and at the same time to preserve virtually all the ligaments.

\section{Surgical Procedure}

This patient (-Fig. 1) is an appropriate candidate for preservation rhinoplasty; she has a small dorsal hump in both the cartilaginous and bony dorsum and an under-projected tip. We used open approach and preserved all the ligaments.

For the open approach, we use an inverted $\mathbf{V}$-shaped incision. In this patient, we have not planned changes on the nasal dorsum and there is no need for rasping of camouflage which is why we continued our dissection with the ligament preservation method and no skin and superficial muscular aponeurotic system (SMAS) dissection on the dorsum. During skin and SMAS dissection, we created a subperiostal tunnel for lateral and transverse osteotomies by approaching the piriform aperture laterally from the vertical scroll ligament, while preserving both the Pitanguy's and scroll ligaments and not elevating the nasal skin (-Figs. 2, 3).

As osteotomy is conducted by Piezoelectric device, a tunnel was created wide enough to enable using the device.

We reached the septum from hemitransfixion incision via subperichondrial dissection. Then we performed a septal cartilage resection in the amount of planned hump reduction using surgical blade and dissection scissors. With an incision right underneath the dorsal hump, prolongated up to the meeting point of ethmoid bone and perpendicular plate, we removed the strip of septal cartilage by separating it from the ethmoid bone and preserving the caudal part of septal cartilage. The bony part of the septum was reduced by excision of the upper part of the perpendicular plate with the help of $2 \mathrm{~mm}$ Baby Rongeur (Ada Medical Instruments) (-Fig. 4). Removal of cartilageous and bony septal strips disarticulate junction between septum and the dorsum.

Additionally, we performed triangular shape bone excision in the piriform aperture (Webster triangle) to avoid possible blockage by inferior turbinate while shifting the bones down (-Fig. 5).

Lateral osteotomy was performed sagittally with Piezo straight saw insert on the level of facial groove (-Fig. $\mathbf{6}$ ). In this case, lateral osteotomy conducted in the sagittal plane makes it possible to easily push down the nasal dorsum, in both osteotomies and osteotomies. Moreover, as the borders of the new bones are parallel to the sagittal plane, it enables the dorsum to move lower and prevents complications, such as residual hump or hump recurrence.

For transverse osteotomies, we used Tastan-Cakir saws on the sides (-Fig. 7).
Issue Theme New Tools and Proven Techniques in Rhinoplasty; Guest Editors: Enrico Robotti, MD, and Werner J. Heppt, MD
Copyright (C) 2019 by Thieme Medical Publishers, Inc., 333 Seventh Avenue, New York, NY 10001, USA. Tel: +1(212) 584-4662.
DOI https://doi.org/ 10.1055/s-0039-1678578. ISSN $0736-6825$. 


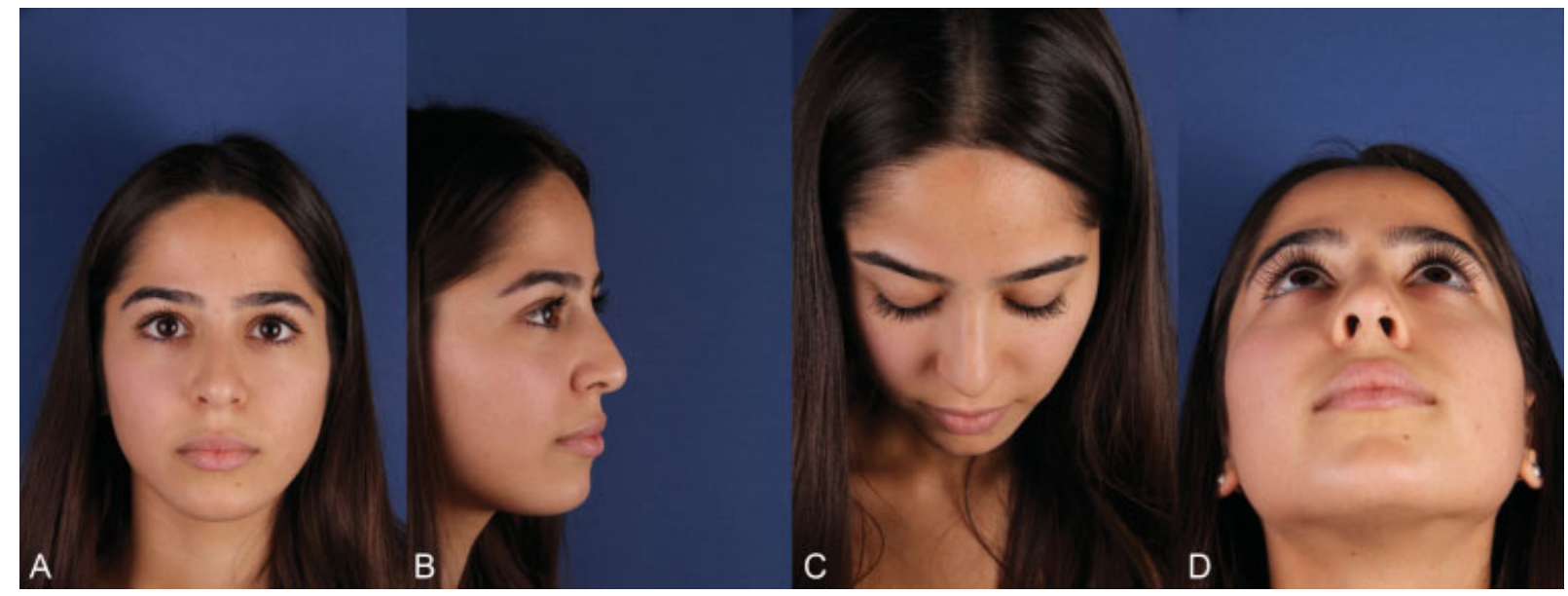

Fig. 1 (A-D) Different views of a patient appropriate for preservation rhinoplasty.

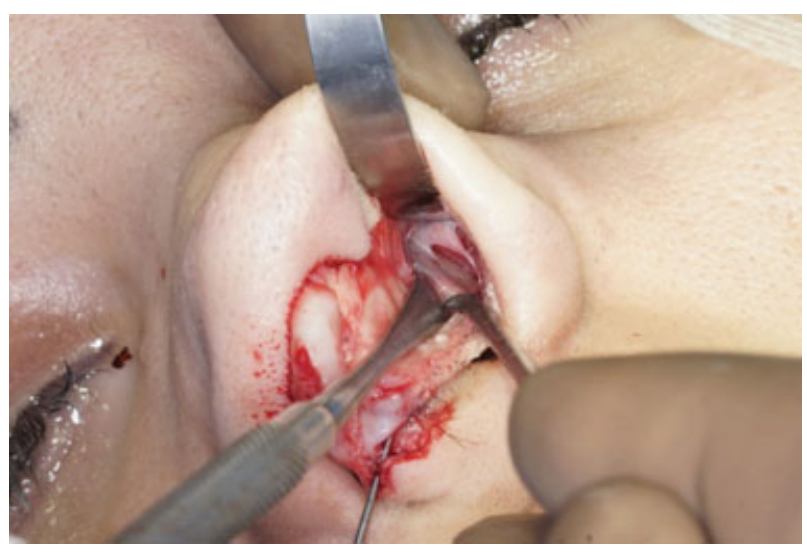

Fig. 2 Subperiostal tunnel for lateral and transverse osteotomies.

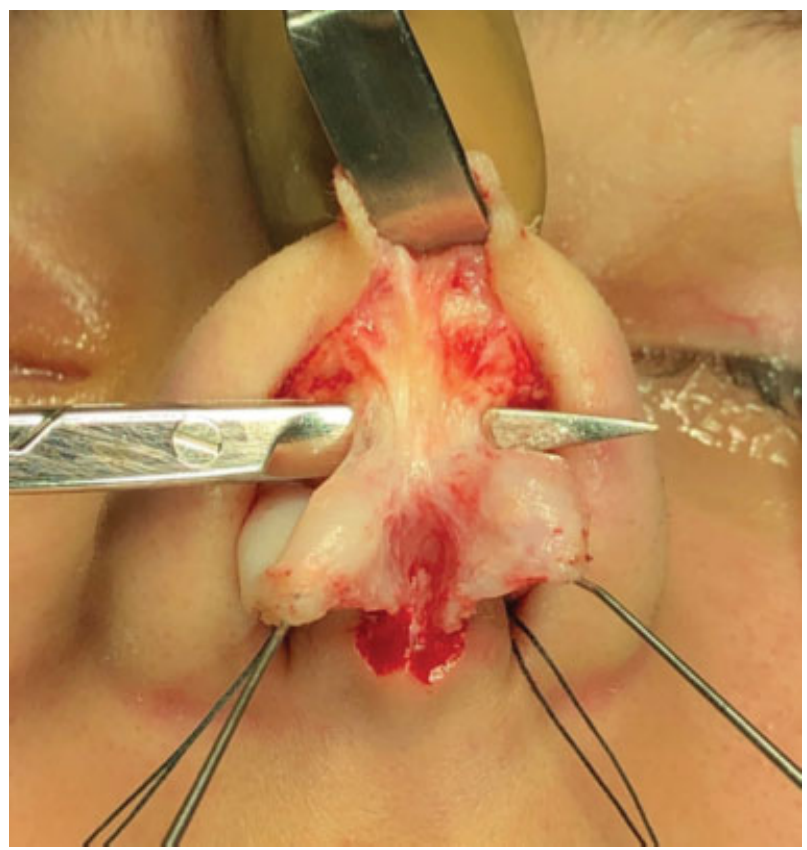

Fig. 3 Intact Pitanguy's ligament.

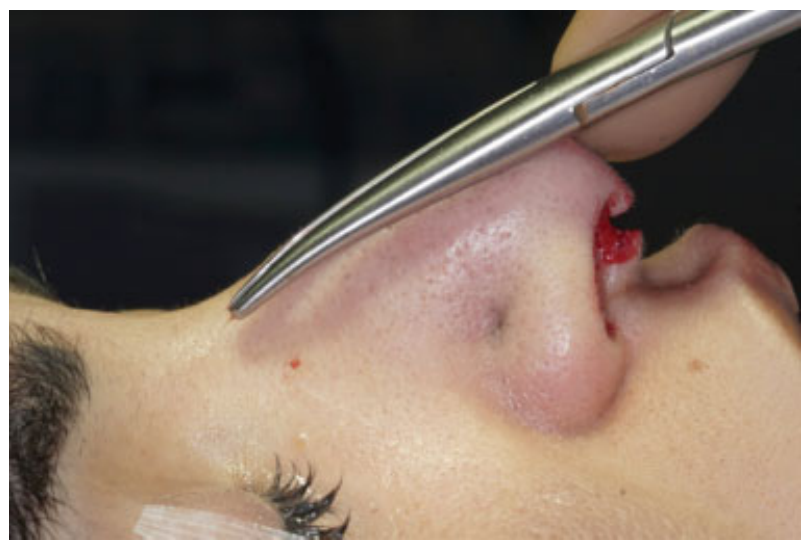

Fig. 4 Bony septum was excised by using $2 \mathrm{~mm}$ Baby Rongeur.

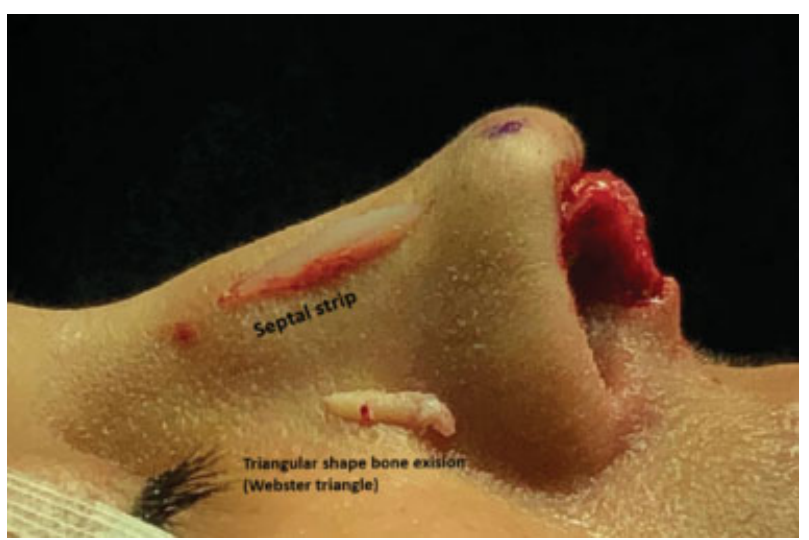

Fig. 5 Excised septal strip and triangular shape bone excision in the piriform aperture (Webster triangle).

For connecting both sides of transverse osteotomies additional incision was performed externally using the $2 \mathrm{~mm}$ osteotome (-Fig. 8).

After finishing precise osteotomies we elevated the periosteum of the inner surface of the maxillary bone, thereby preventing the tissue resistance and creating the space for shifting the dorsum down freely (-Fig. 9). 


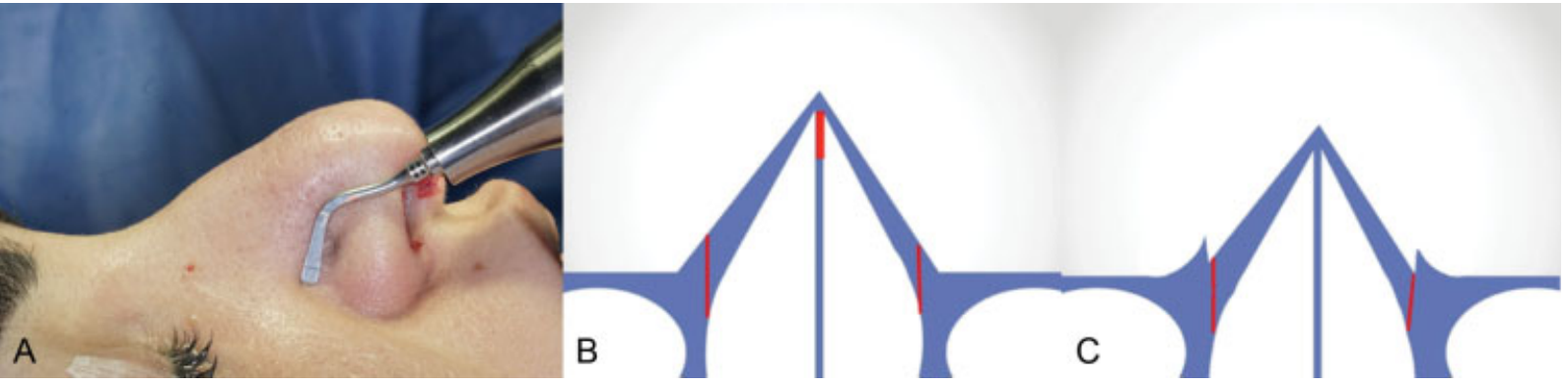

Fig. 6 (A-C) Sagittal osteotomy with Piezo straight saw insert.

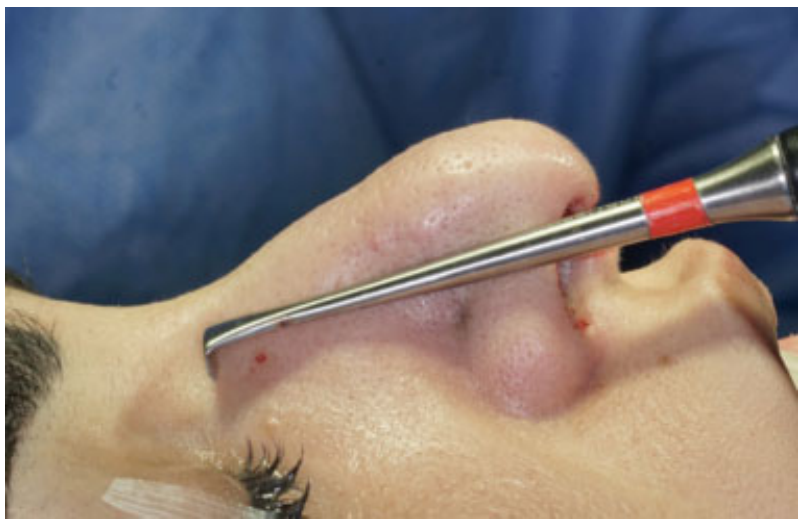

Fig. 7 The Tastan-Cakir saw.

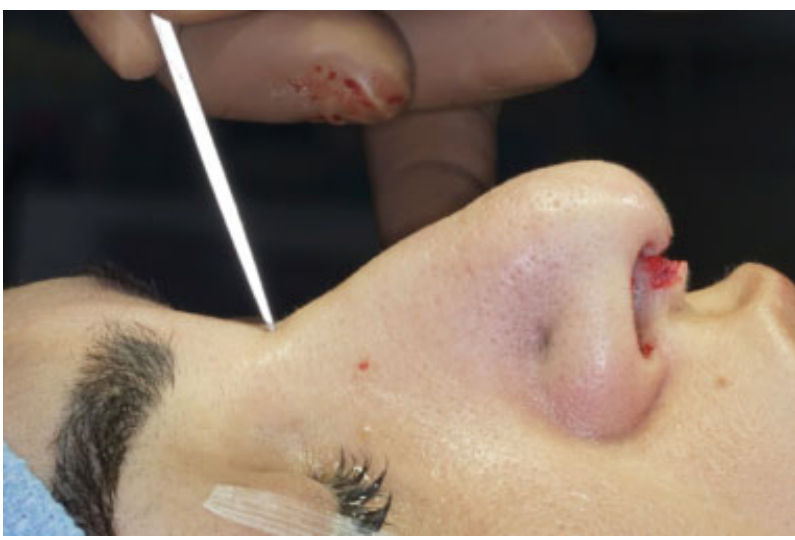

Fig. 8 External incision with $2 \mathrm{~mm}$ osteotome.

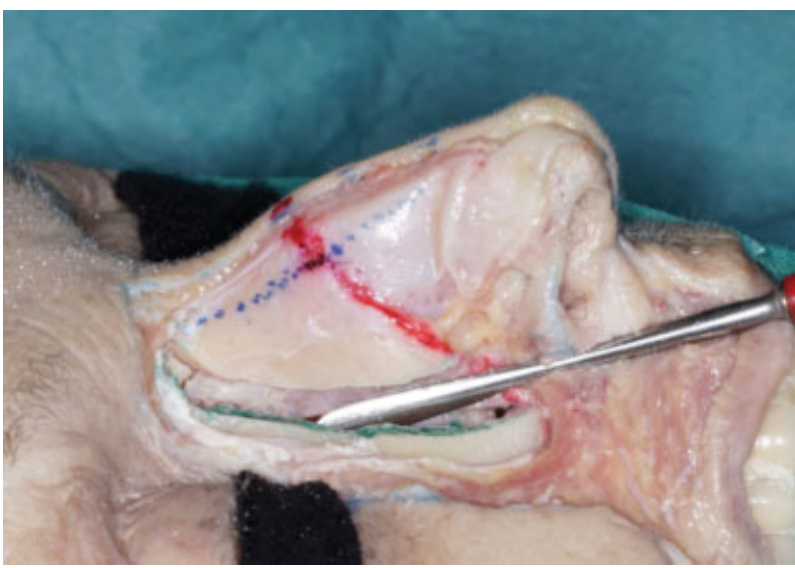

Fig. 9 Periosteum elevation of the inner surface of the maxillary bone (demonstrated on cadaver).

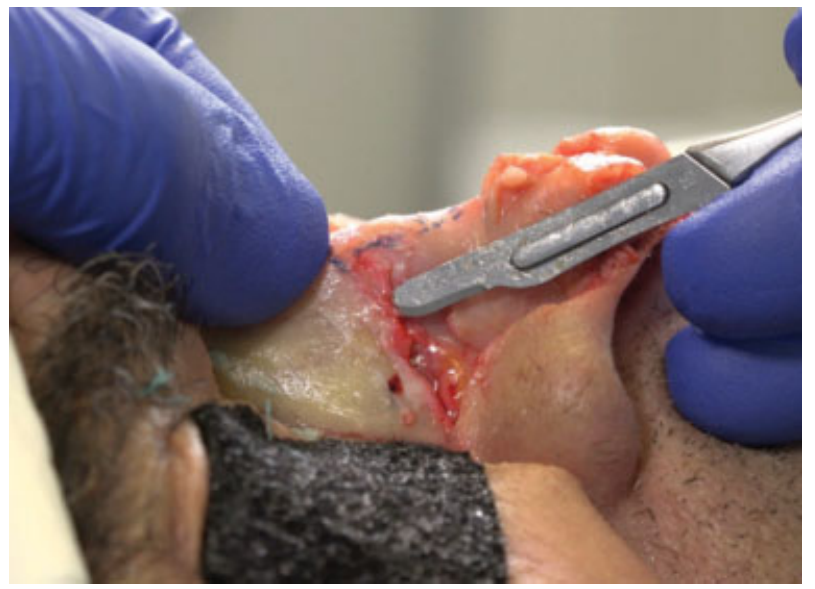

Fig. 10 Lateral dissection: undermining upper lateral cartilage from the nasal bone in the key-stone area (demonstrated on cadaver).

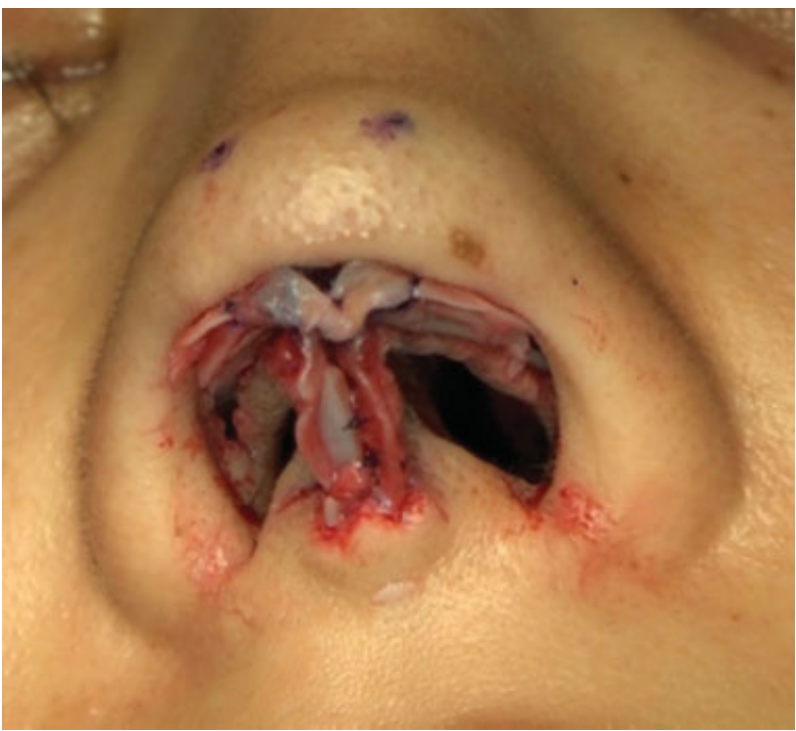

Fig. 11 Double dome graft.

The next maneuver is lateral dissection between upper lateral cartilage and nasal bones (-Fig. 10). As the nasal dorsal hump is not a two-dimensional structure apart from the release at the dorsal keystone area we should also mobilize the lateral keystone area to change the shape of the nasal dorsal anatomy. With this maneuver, as the side wall connections will be mobilized and there is no areas to resist the newly shaped nasal dorsum, hump recurrence problem will also be eliminated. 
Without this dissection, the dorsum would only drop down without changing the shape, which doesn't match our goal of not only lowering but also straightening the dorsum.

The remnant of the septal cartilage just immediately under the dorsal key-stone area was scored for adding flexibility. When all those maneuvers were completed, dorsal hump was pushed downwards and reshaped.

For safe preservation hump reduction it's crucial to follow this steps sequentially:

1. Septal cartilage resection and separation both bony and cartilageous parts of the septum from the dorsum for avoiding unwanted fractures of the septum during push down process.
2. Triangular shape bone excision in the piriform aperture (Webster triangle) to avoid blockage by inferior turbinate while shifting the bones down.

3. Elevation the periosteum of the inner surface of the maxillary bone releasing the tissue resistance.

4. Lateral dissection (undermining upper lateral cartilage from the nasal bones) to enable straightening the dorsum during push down.

5. The remnant cartilage scoring under the dorsal key-stone area.

After finishing all manipulations on the nasal dorsum, we moved to the nose tip reshaping, starting from

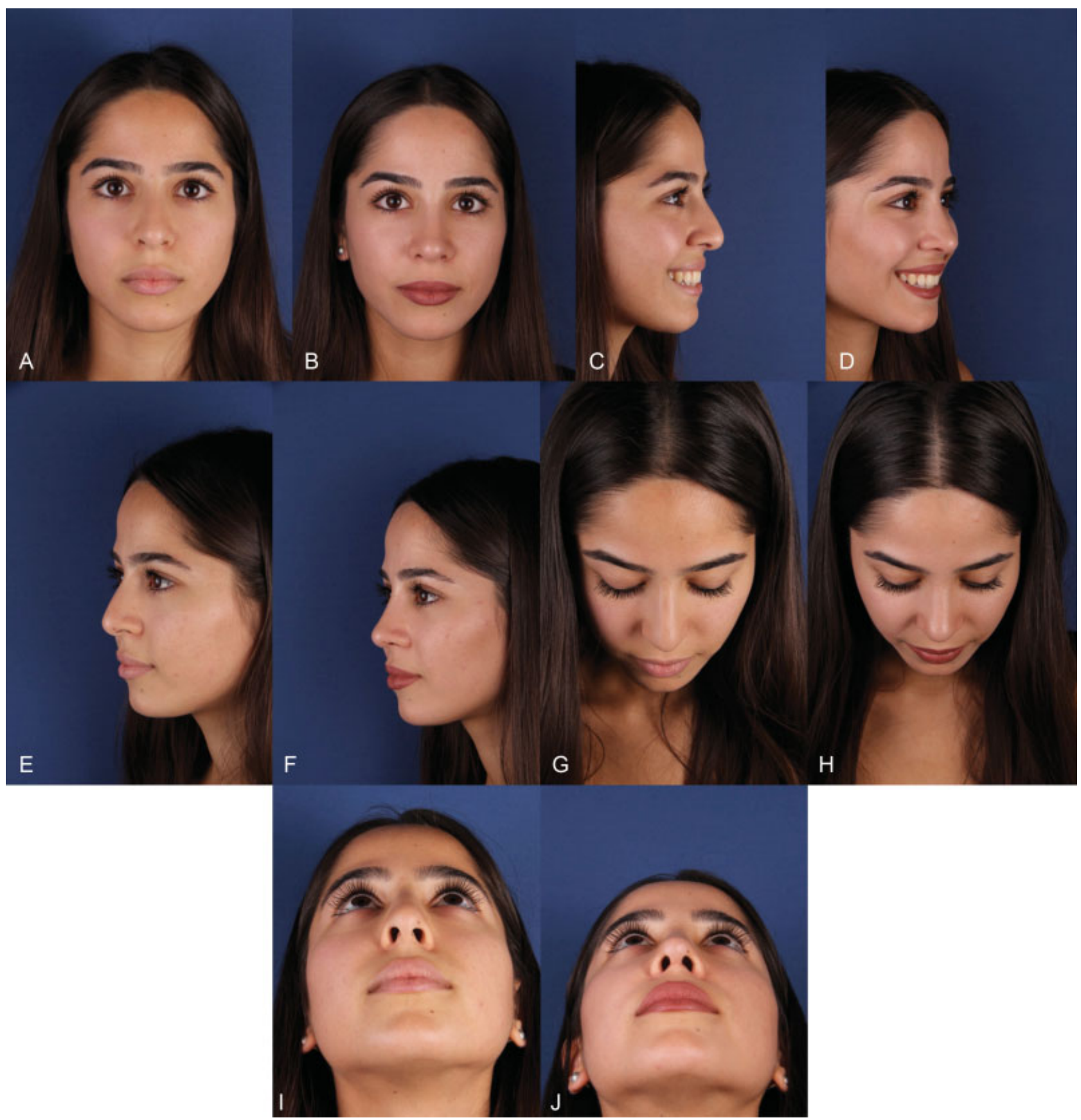

Fig. 12 Result 6 months after open Piezo preservation rhinoplasty. (A, C, E, G, I) Preoperatively, (B, D, F, H, J) postoperatively. 
supraperichondreal dissection of the lower lateral cartilages through the columellar inverted V-shaped incision. We dissected lateral crura from vestibular skin and made them free. We used lateral crural strut graft for crura flattening and shaping. Then we placed those grafts into the pocket created in the direction toward the lateral canthus and fixed them with 5.0 Vicryl (Ethicon, Inc.). We created additional support and equalized the heights of both medial crura by using columellar strut graft, placed as posteriorly as possible. Both sides of the lateral crura were attached with the sutures passing through the skin, helping to fix the positioning of the crura and to model crural shapes more accurately.

On the tip, we used cranial tip sutures, adding for safety a corner stitch after cutting out dog ear. To get a better light reflection on the lateral crura's caudal margin, we turned in 1 to $2 \mathrm{~mm}$ cartilage by cutting with a 15 blade on the both sides. Then the resected portions of the lateral crura were reattached on both domes separately (double dome graft), resulting in better and more natural light reflection on the nose tip and higher tip projection (-Fig. 11).

We covered septal cartilage with the airway silicon Doyle's splints. After completing the tipplasty, we closed columellar incision with 6.0 polydioxanone sutures by putting at least 5 knots on every corner. Marginal incisions were sutured with 6.0 rapid Vicryl.

For the dissection zone created for lateral osteotomies and entered subperiostally, we placed drains prepared from venous catheters to stay for 1 day.

We put first a thin layer of the cotton pad on bandaging stage to prevent any pulling during removal of the plaster. Additionally, we placed one layer of sponge for extra stabilization of the new shape by pressing on the nose dorsum. - Figs 12 show the result of the presented patient after 6 months.

\section{Discussion}

The idea of performing a rhinoplasty with correction of the dorsal hump at the same time preserving the nasal dorsum is both aesthetically and functionally more adequate and reduces the operation time and graft material requirements considerably. Further, recovery time is also reduced by means of preservation technique due to intact skin and SMAS attachments in the nasal dorsum area.

We performed Dr. Saban's modified push-down technique for preserving the nasal dorsum, by using this technique from the open approach with the assistance of Piezoelectric instrument. Piezoelectric device does not damage soft tissues and membranes, nor does it cause bleeding during the bone shaping process. Owing to using Piezoelectric device in this case there was no significant bruising and swelling. Only slight swelling was noticed on the 3rd and 4th day after the surgery.

Open approach in turn gives better visuality for tip reshaping. With this open preservation approach, tip is getting more flexible and the dorsal aesthetic lines look more natural.

In this case, combined application of preservation method, open approach and usage of Piezoelectric device allowed us to achieve good and natural result.

Conflict of Interest

None. 